\title{
Incidencia de complicaciones de la cirugía de extracción del lentículo por incisión pequeña (SMILE)
}

\section{Incidence of complications of Small Incision Lenticule Extraction (SMILE)}

\author{
Samuel J. Avalos-Lara*, Cristina Pacheco-del Valle, Oscar Baca-Lozada, Elisa D. Alegría-Gómez, \\ Oscar Fernández-Vizcaya y Regina Velasco-Ramos \\ Departamento de Alta Especialidad en Córnea y Cirugía Refractiva, Fundación Hospital Nuestra Señora de la Luz, IAP, Ciudad de México, México
}

\section{Resumen}

Objetivo: Conocer las complicaciones y la incidencia en la curva de aprendizaje de la técnica quirúrgica de extracción del lentículo por incisión pequeña (SMILE). Material y métodos: Estudio prospectivo, descriptivo y observacional. Se estudiaron las complicaciones intraoperatorias y postoperatorias de los pacientes sometidos a cirugía refractiva con la técnica quirúrgica de SMILE por femtosegundo, de enero de 2015 a enero de 2018, en la Fundación Hospital Nuestra Señora de la Luz. Para la cirugía se utilizó la plataforma VisuMax ${ }^{\circledR}$ de Zeiss ${ }^{\circledR}$. Se realizó el análisis estadístico en la plataforma Excel. Resultados: Se estudiaron 526 ojos de 263 pacientes sometidos a cirugía refractiva con técnica quirúrgica SMILE. Las complicaciones transoperatorias se observaron en 70 de los 526 ojos. La principal complicación fue dificultad para extraer el lentículo, 27 ojos (5.13\%), seguido de abrasión epitelial, 15 ojos (2.85\%), desgarro de la incisión, 12 ojos (2.28\%), pérdida de la succión, 7 ojos (1.33\%), ruptura del lentículo, 4 ojos (0.76\%), black spot, 4 ojos (0.76\%) y hemorragia en interfase, 1 ojo (0.19\%). Todas las complicaciones se resolvieron de manera transoperatoria. Conclusiones: La técnica quirúrgica SMILE es segura, con excelentes resultados en la agudeza visual final.

Palabras clave: Cirugía refractiva. SMILE. Complicaciones.

\section{Summary}

Purpose: To summarize the complications and their incidence in the learning curve of the "small incision lenticule extraction (SMILE)"surgical technique. Material and methods: Prospective, descriptive and observational study. We evaluated the intraoperative and postoperative complications of patients undergoing SMILE refractive surgery with femtosecond laser from January 2015 to January 2018 at the Hospital Nuestra Señora de la Luz, Mexico. The platform used for surgery was the VisuMax by Zeiss. Statistical analysis was performed with Excel. Results: 526 eyes of 263 patients undergoing "SMILE" refractive surgery were studied. Intraoperative complications were observed in 70 of 526 eyes. The main complication was difficult lenticular extraction in 27 eyes (5.13\%), epithelial abrasion in 15 eyes (2.85\%), incision tear 12 in eyes (2.28\%), lost vacuum in 7 eyes (1.33\%), incomplete lenticule removal in 4 eyes (0.76\%), black spot in 4 eyes (0.76\%) and wound bleeding in 1 eye (0.19\%). All complications were resolved intraoperatively. Conclusions: SMILE surgical technique is safe, showing excellent results in final visual acuity.

Key words: Refractive surgery. Small incision lenticule extraction. Complications.

\section{Correspondencia:}

*Samuel J. Avalos-Lara

Ezequiel Montes, 135

Col. Tabacalera, Del. Alcaldía Cuauhtémoc Fecha de recepción: 28-06-2018

C.P. 06030, Ciudad de México, México Fecha de aceptación: 16-12-2019

E-mail: josue.27al@gmail.com

DOI: 10.24875/RMO.M20000109

Disponible en internet: 01-03-2020 Rev Mex Oftalmol. 2020;94(2):83-89 www.rmo.com.mx 0187-4519/@ 2019 Sociedad Mexicana de Oftalmología. Publicado por Permanyer. Este es un artículo open access bajo la licencia CC BY-NC-ND (http://creativecommons.org/licenses/by-nc-nd/4.0/). 


\section{Introducción}

La cirugía refractiva es un proceso electivo donde el paciente busca mejorar su visión mediante procedimientos quirúrgicos ${ }^{1}$. La cirugía de extracción del lentículo por incisión pequeña (SMILE, por sus siglas en inglés, small incision lenticle extraction) consiste en crear un lentículo dentro de una córnea intacta, y posteriormente removerla mediante una pequeña incisión, típicamente menor a $4 \mathrm{~mm}$ de tamaño². La cirugía de SMILE ofrece la ventaja de que no requiere hacer un colgajo (flap), por lo que crea una mínima disrupción de la arquitectura del estroma anterior ${ }^{3}$.

Se requiere del láser femtosegundo VisuMax ${ }^{\circledR}$ (Carl Zeiss Meditec, Jena, Germany), el cual fue introducido en el año $2007^{4}$. La interfase posterior del estroma lenticular es creada primero usando una dirección de periferia al centro (out-to-in), seguida de la interfase anterior del lentículo, usando una dirección del centro a la periferia (in-to-out). Finalmente se realiza una incisión de 2 a $4 \mathrm{~mm}$, usualmente en el cuadrante superotemporal. Posteriormente, bajo microscopio se lleva a cabo la separación y extracción del lentículo ${ }^{5}$.

Son muy pocos los estudios en los que se reportan complicaciones relacionadas con la cirugía SMILE. Ivarsen, et al. realizaron un estudio donde se realizaron 1,800 procedimientos SMILE. Las complicaciones transoperatorias más frecuentes fueron: abrasiones epiteliales, dificultades de la extracción del lentículo y desgarros pequeños de la incisión, mientras que las complicaciones postoperatorias más frecuentes fueron haze corneal, inflamación de interface, irregularidad de la topografía corneal ${ }^{6}$. Al ser una técnica que únicamente afecta el estroma posterior, hay una mayor preservación del plexo nervioso subbasal corneal, lo que disminuye el riesgo de presentar ojo seco como complicación postoperatoria a largo plazo 7 . Otra complicación reportada es el residuo lenticular que produce astigmatismo irregular, que puede ser tratada eficazmente usando queratectomía epitelial fototerapéutica (PTK) $)^{8,9}$.

La cirugía SMILE actualmente está limitada para usarse en pacientes con miopía de hasta -10.00 dioptrías con errores cilíndricos de hasta -6.00 dioptrías ${ }^{10}$. Sin embargo, esta cirugía ha demostrado que no existen cambios significativos en el equivalente esférico tras 5 años de seguimiento ${ }^{11}$. La cirugía SMILE ha demostrado resultados cercanamente idénticos a los obtenidos con cirugía LASIK (Laser Assisted in Sltu Keratomileusis, por sus siglas en inglés) ${ }^{12}$.

\section{Objetivo}

El objetivo de este estudio es conocer las complicaciones transoperatorias y postoperatorias presentadas en pacientes sometidos a cirugía electiva refractiva tipo SMILE, así como su incidencia. Asimismo, comparar la incidencia de complicaciones transoperatorias y postoperatorias que se presentan en la Fundación Hospital Nuestra Señora de la Luz con la de la literatura.

\section{Métodos}

Se llevó a cabo un estudio prospectivo, descriptivo y longitudinal. Se estudiaron las complicaciones transoperatorias y postoperatorias de los pacientes sometidos a cirugía refractiva con técnica quirúrgica de SMILE por femtosegundo, de enero de 2015 a enero de 2018, en el Departamento de Córnea y Cirugía Refractiva de la Fundación Hospital Nuestra Señora de la Luz. La plataforma para la cirugía es VisuMax ${ }^{\circledR}$ de Zeiss ${ }^{\circledR}$. Se incluyeron pacientes mayores de 18 años, pacientes sin patología ocular, topografía corneal normal, espesor corneal mayor a 500 micras y defecto refractivo miópico simple o astigmatismo miópico compuesto. La técnica quirúrgica se realizó con anestesia tópica, se creó un lentículo con diámetro de $6.5 \mathrm{~mm}$ a una profundidad de 120 micras y una incisión corneal de $4 \mathrm{~mm}$. El lentículo se disecó y posteriormente se retiró, se irrigó con solución salina balanceada para extraer detritus y finalmente se aplicó antibiótico (gatifloxacino $0.3 \%$ ) y antiinflamatorio esteroideo (prednisolona 1\%). Se comparó la incidencia de complicaciones presentadas durante tres años consecutivos con la reportada en la literatura.

\section{Resultados}

Se incluyeron 526 ojos de 263 pacientes. Las complicaciones transoperatorias se observaron en 70 de los 526 ojos. La principal complicación fue dificultad para extraer el lentículo, 27 ojos (5.13\%), seguida de abrasión epitelial, 15 ojos (2.85\%), desgarro de la incisión, 12 ojos (2.28\%), pérdida de la succión, 7 ojos (1.33\%), ruptura del lentículo, 4 ojos $(0.76 \%)$, black spot, 4 ojos $(0.76 \%)$ y hemorragia en interfase, 1 ojo $(0.19 \%)$ (Tabla 1 y Fig. 1). Todas las complicaciones se resolvieron de manera transoperatoria. En cuanto a los pacientes con pérdida de la succión, en dos de ellos se realizó incisión de manera manual, en los 5 ojos restantes se reprogramó su cirugía refractiva 3 meses después.

En 122 ojos se demostraron hallazgos postquirúrgicos mediante biomicroscopia desde el día 1 hasta el 
Tabla 1. Muestra las complicaciones transoperatorias con el porcentaje de incidencia de acuerdo al número de complicaciones (n) y el porcentaje del total (\%) de los casos

\begin{tabular}{|l|c|c|}
\hline Complicaciones, $(\mathbf{n = 5 2 6})$ & $\mathrm{n}$ & $\%$ \\
\hline Dificultad para extraer el lentículo & 27 & $5.13 \%$ \\
\hline Abrasión epitelial & 15 & $2.85 \%$ \\
\hline Desgarro de la incisión & 12 & $2.28 \%$ \\
\hline Pérdida de la succión & 7 & $1.33 \%$ \\
\hline Ruptura del lentículo & 4 & $0.76 \%$ \\
\hline Black spot & 4 & $0.76 \%$ \\
\hline Hemorragia interfase & 1 & $0.19 \%$ \\
\hline
\end{tabular}

Tabla 2. Muestra las complicaciones postoperatorias con el porcentaje de incidencia de acuerdo al número de complicaciones (n) y el porcentaje del total $(\%)$ de los casos

\begin{tabular}{|l|c|c|}
\hline Complicaciones, $(\mathbf{n}=526)$ & n & $\%$ \\
\hline Detritus & 61 & $11.59 \%$ \\
\hline Queratopatía punteada superficial & 27 & $5.13 \%$ \\
\hline Queratitis lamelar difusa & 9 & $1.71 \%$ \\
\hline Defecto epitelial & 8 & $1.52 \%$ \\
\hline Haze & 8 & $1.52 \%$ \\
\hline Edema interfase & 4 & $0.76 \%$ \\
\hline Pliegues en la incisión & 2 & $0.38 \%$ \\
\hline Depósitos numulares & 1 & $0.19 \%$ \\
\hline Crecimiento epitelial & 1 & $0.19 \%$ \\
\hline Lentículo interfase & 1 & $0.19 \%$
\end{tabular}

día 7. Lo más frecuente fue la presencia de detritus, 61 ojos (11.59\%), queratopatía punteada superficial, 27 ojos $(5.13 \%)$, queratitis lamelar difusa, 9 ojos $(1.71 \%)$, defecto epitelial y haze corneal, 8 ojos (1.52\%), edema en interfase, 4 ojos $(0.76 \%)$, pliegues en la incisión, 2 ojos $(0.38 \%)$, depósitos numulares, crecimiento epitelial y lentículo en interfase, un caso por cada uno de ellos $(0.19 \%)$ (Tabla 2$)$.

En las tablas 3 y 4 se resumen las complicaciones transoperatorias y postoperatorias, respectivamente, encontradas en distintas literaturas, incluyendo la incidencia de complicaciones en nuestro hospital.

Los resultados visuales finales se obtuvieron con seguimiento a 2 años en 204 ojos y a 1 año en 322

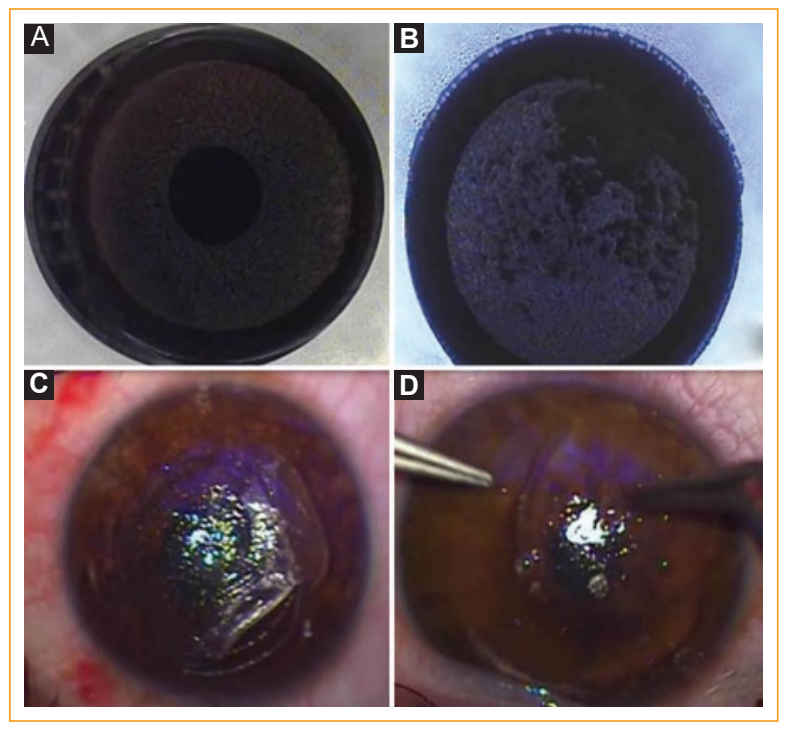

Figura 1. A: Black spot. B: Pérdida de la succión. C: Desgarro de la incisión. D: Lentículo incompleto.

ojos. Las agudezas visuales finales se dividieron en los siguientes grupos: grupo 1 = mejor de 20/40; grupo 2 = 20/50-20/100; grupo $3=$ peor de 20/100.

Se encontró que 524 ojos de 526 (99.61\%) sometidos a cirugía refractiva SMILE pertenecían al grupo 1. De estos, 54 ojos presentaron agudeza visual final de 20/15; 373 ojos, de 20/20; 65 ojos, de 20/25; 22 ojos, de 20/30, y únicamente 7 ojos, de 20/40.

En el grupo 2, únicamente se agruparon 2 ojos de $526(0.38 \%)$ con agudeza visual final de $20 / 50$ y $20 / 80$, respectivamente. En el ojo de agudeza visual de 20/50 se presentó subcorrección de -0.75 esfera con +1.00 de cilindro a $135^{\circ}$. En el ojo de 20/80 el paciente se conocía con ambliopía anisometrópica.

Ninguno de los ojos sometidos a cirugía SMILE se incluyó en el grupo 3 (Tabla 5).

\section{Discusión}

A pesar de ser uno de los estudios en donde más ojos se incluyeron (3,004 ojos), Wang, et al. reportan una incidencia muy baja de complicaciones transoperatorias, probablemente debido a que los procedimientos se realizaron por un solo cirujano experimentado, a diferencia de nuestra institución donde los oftalmólogos en formación también realizan este tipo de procedimiento ${ }^{13}$. La pérdida de la succión apenas se presentó en 28 casos $(0.93 \%)$, y fue más frecuente durante la creación del plano anterior. La incidencia puede aumentar debido al nivel de ansiedad que presenta el paciente ${ }^{14}$. 
Tabla 3. Complicaciones transoperatorias de la cirugía refractiva SMILE comparado con la literatura

\begin{tabular}{|c|c|c|c|c|c|c|c|}
\hline & $\begin{array}{l}\text { Hospital } \\
\text { de la Luz } \\
\text { (526 ojos) }\end{array}$ & $\begin{array}{c}\text { Ivarsen, } \\
\text { et al. }{ }^{6} \\
(1,574 \text { ojos })\end{array}$ & $\begin{array}{c}\text { Ramírez, } \\
\text { et al. }{ }^{17} \\
\text { (160 ojos) }\end{array}$ & $\begin{array}{c}\text { Chee, } \\
\text { et al. }{ }^{14} \\
\text { (340 ojos) }\end{array}$ & $\begin{array}{c}\text { Osman, } \\
\text { et al. }{ }^{15} \\
(3,376 \text { ojos })\end{array}$ & $\begin{array}{c}\text { Wang, } \\
\text { et al. }{ }^{13} \\
(3,004 \text { ojos })\end{array}$ & $\begin{array}{c}\text { Chansue, } \\
\text { et al. }{ }^{2} \\
\text { (347 ojos) }\end{array}$ \\
\hline Dificultad de la extracción del lentículo & $5.13 \%$ & $1.9 \%$ & - & - & - & - & - \\
\hline Desgarros en la incisión & $2.28 \%$ & $1.8 \%$ & $16.2 \%$ & - & - & $0.17 \%$ & - \\
\hline Abrasiones epiteliales & $2.85 \%$ & $6 \%$ & - & - & - & - & - \\
\hline Pérdida de la succión & $1.33 \%$ & $0.8 \%$ & $11.6 \%$ & $4.4 \%$ & $2.1 \%$ & $0.93 \%$ & - \\
\hline Black spot & $0.76 \%$ & - & $14 \%$ & - & - & $0.33 \%$ & - \\
\hline Ruptura del lentículo & $0.76 \%$ & - & - & - & - & $0.27 \%$ & - \\
\hline Hemorragia en interfase & $0.19 \%$ & - & - & - & - & $0.93 \%$ & - \\
\hline $\begin{array}{l}\text { Perforación de la cara anterior de la } \\
\text { córnea }\end{array}$ & - & $0.22 \%$ & - & - & - & - & - \\
\hline $\begin{array}{l}\text { Capa de burbuja opaca que impide } \\
\text { realizar la incisión }\end{array}$ & - & - & $16.2 \%$ & - & - & $1.73 \%$ & - \\
\hline
\end{tabular}

Tabla 4. Complicaciones postoperatorias de la cirugía refractiva SMILE comparado con la literatura

\begin{tabular}{|c|c|c|c|c|c|}
\hline & $\begin{array}{l}\text { Hospital de la Luz } \\
\text { (526 ojos) }\end{array}$ & $\begin{array}{c}\text { Ivarsen, et al. }{ }^{6} \\
(1,574 \text { ojos })\end{array}$ & $\begin{array}{c}\text { Ramírez, et al. }{ }^{17} \\
\text { (160 ojos) }\end{array}$ & $\begin{array}{c}\text { Zhao, et al. }{ }^{20} \\
(1,112 \text { ojos })\end{array}$ & $\begin{array}{l}\text { Qiu, et al. }{ }^{22} \\
\text { (193 ojos) }\end{array}$ \\
\hline Detritus & $11.59 \%$ & - & - & - & - \\
\hline Queratopatía punteada superficial & $5.13 \%$ & - & - & - & - \\
\hline Haze corneal & $1.52 \%$ & $8 \%$ & - & - & - \\
\hline Defecto epitelial & $1.52 \%$ & - & $41.9 \%$ & - & - \\
\hline Disfunción de la película lagrimal & - & $5 \%$ & - & - & $54 \%$ \\
\hline Edema de interface & $0.76 \%$ & $0.3 \%$ & - & - & - \\
\hline Queratitis lamelar difusa & $1.71 \%$ & - & - & $1.6 \%$ & - \\
\hline Residuo lenticular & $0.19 \%$ & - & - & - & - \\
\hline
\end{tabular}

SMILE: extracción del lentículo por incisión pequeña.

En otras series, la incidencia de pérdida de la succión por cirugía SMILE es de 2.1 a $4.4 \%$. Su incidencia disminuye con la experiencia adquirida del cirujano en la técnica ${ }^{14,15}$. Puede ocurrir como resultado de diversos factores, como movimientos repentinos del ojo, forzamiento de los párpados durante la aplicación del láser, ingreso de fluido entre el lente de contacto y la córnea, migración de gas y fuerzas compresivas contra el lente de contacto.

Ivarsen, et al (2014) reportan como complicación transoperatoria más frecuente: abrasión epitelial (6\%), dificultad de extracción del lentículo $(1.9 \%)$ y desgarro de la incisión $(1.8 \%)^{16}$. En nuestro estudio, las complicaciones transoperatorias más frecuentes fueron, en primer lugar, dificultad de extracción del lentículo (5.13\%), seguido de abrasión epitelial (2.85\%) y desgarro de la incisión (2.28\%). Esta diferencia se explica probablemente por la curva de aprendizaje de esta nueva técnica quirúrgica, ya que en nuestro medio se cuenta con apenas 4 años de experiencia de realizarla.

En comparación con otro centro oftalmológico de México, Ramírez, et al (2015) reportan mayor incidencia en complicaciones, y las de mayor frecuencia fueron defecto epitelial (41.9\%), desgarro de la incisión $(16.2 \%)$ y black spot $(14 \%)^{17}$. En el Hospital de la Luz, la incidencia no se correlaciona, presentando en menor incidencia el defecto epitelial (1.52\%), desgarro de la incisión $(2.28 \%)$ y black spot $(0.76 \%)$. A pesar de 
Tabla 5. Agudeza visual final de pacientes sometidos a cirugía SMILE con seguimiento de 2 y 1 año

\begin{tabular}{|l|c|c|}
\hline Grupo & Agudeza visual final & $n(\%)$ \\
\hline $\begin{array}{l}\text { Grupo } 1 \\
\text { Mejor de 20/40 } \\
(99.61 \%)\end{array}$ & $20 / 15$ & $54(10.26)$ \\
\hline & $20 / 20$ & $373(70.91)$ \\
\hline $\begin{array}{l}\text { Grupo 2 } \\
\text { 20/50-20/100 }\end{array}$ & $20 / 25$ & $65(12.35)$ \\
$(0.38 \%)$ & $20 / 30$ & $22(4.18)$ \\
\hline $\begin{array}{l}\text { Grupo 3 } \\
\text { Peor de 20/100 } \\
(0 \%)\end{array}$ & $20 / 50$ & $1(1.33)$ \\
\hline
\end{tabular}

SMILE: extracción del lentículo por incisión pequeña.

presentar una alta incidencia en defecto epitelial, se trata de una complicación transitoria que no repercute en la agudeza visual. Probablemente, en nuestra institución se tiene una menor incidencia en el desgarro de incisión porque se realiza una incisión ligeramente más grande para retirar el lentículo.

La mayoría de las complicaciones transoperatorias de la cirugía se deben a la inexperiencia del cirujano para reconocer el borde del lentículo. Esto obliga al cirujano a seguir tratando de disecar debajo o encima del lentículo por un periodo prolongado, lo que lleva al aumento en la posibilidad de complicaciones como rotura de la incisión o del lentículo, así como mayor tiempo de cirugía. El reconocimiento apropiado del borde del lentículo facilita la cirugía debido al reconocimiento rápido del lentículo con sus superficies anterior y posterior, acortando así la duración operativa media y minimizando las complicaciones transoperatorias ${ }^{18}$.

Las complicaciones postoperatorias más frecuentes fueron detritus (11.59\%), queratopatía punteada superficial $(5.13 \%)$, defecto epitelial y haze (1.52\%). Ivarsen, et al. reportan el haze corneal como complicación postoperatoria más frecuente (8\%). Las complicaciones postoperatorias son publicadas en mucho menor medida que las transoperatorias. La mayoría de los autores no toman en cuenta el detritus como una complicación postoperatoria, ya que no repercute en la agudeza visual.

El residuo lenticular se presentó solamente en 1 paciente $(0.19 \%)$. Encontramos solamente un reporte de caso publicado en donde se reporta residuo lenticular por tomografía por coherencia óptica (OCT) 5 meses después del procedimiento quirúrgico. Presentó pérdida de visión de 3 líneas de visión 1 mes después de la operación y, posteriormente, mejoría de la visión de 2 líneas de visión 5 meses después de la operación ${ }^{19}$.

La queratitis lamelar difusa fue descrita inicialmente como una complicación temprana por cirugía LASIK, con una incidencia de 0.13 a $18.9 \%^{20}$. En el estudio realizado por Zhao, et al., en donde se incluyeron 1,112 ojos de 590 pacientes, se encontró que 18 ojos de 11 pacientes desarrollaron queratitis lamelar difusa, y se reportó una incidencia del $1.6 \%$. En el $77.8 \%$ de los casos fue bilateral y en el $22.2 \%$ fue unilateral. La incidencia se asocia a un mayor diámetro lenticular y lentículos más delgados, lo que resulta en una base estromal residual más gruesa ${ }^{21}$. En nuestro caso, la incidencia fue discretamente mayor (3.99\%), y no es posible asociarla a cambios de diámetro lenticular, ya que todos los pacientes que fueron sometidos al procedimiento tuvieron el mismo diámetro lenticular. De nuestros pacientes que presentaron esta complicación, únicamente uno fue sometido a lavado de interfase en el postoperatorio debido a que presentaba un estadio 3 , posteriormente se manejó con esteroide tópico. La agudeza visual final de este caso fue de 20/25. Todos los demás casos de queratitis lamelar difusa fueron de estadio 1 y 2 , los cuales fueron manejados únicamente con esteroides tópicos y presentaron agudezas visuales finales mejores a 20/25.

Aunque la cirugía SMILE se realiza sin la creación de un flap, y teóricamente de esta forma se mantiene la estructura anatómica y biomecánica de la córnea, algunos pacientes presentan síntomas de ojo seco. Qiu, et al. reportan que el $54 \%$ de los pacientes sometidos a cirugía SMILE presentan síntomas de ojo seco a 1 semana del postoperatorio, sin embargo, a los 3 meses del postoperatorio, los síntomas disminuyeron hasta casi el mismo nivel que en el preoperatorio ${ }^{22}$. En el presente estudio no se estudió la función de película lagrimal.

Las capas de burbujas opacas no se presentaron en nuestros pacientes, sin embargo, Wang, et al. reportan una incidencia del $0.73 \%$. Puede dificultar la cirugía debido a una visualización inadecuada de la disección del lentículo, lo que dificulta al mismo tiempo su extracción, o puede incluso imposibilitarla ${ }^{13}$.

No se presentaron complicaciones infecciosas de ningún origen en nuestro hospital, hay reportes de casos donde se demuestra queratitis intersticial por Streptococcus pneumoniae en ambos ojos ${ }^{23}$. No existe incidencia reportada de queratitis infecciosa por cirugía SMILE en la literatura a conocimiento de los autores, 
sin embargo, en comparación con otras técnicas, la incidencia reportada después de la cirugía LASIK es apenas de $0.03 \%^{24}$.

Los detritus no son considerados una complicación postoperatoria en ninguna de las series publicadas en la literatura. Son consideradas más un hallazgo postoperatorio y no afectan el resultado final del procedimiento refractivo. Al no tomar en cuenta los detritus como una complicación postoperatoria, el porcentaje de complicaciones postoperatoria es apenas del $13.11 \%$, en donde la queratopatía punteada superficial tiene el primer lugar como complicación postoperatoria y puede ser manejada efectivamente con lubricantes oculares.

De los pacientes sometidos a la cirugía refractiva tipo SMILE, el $99.61 \%$ de los pacientes tuvo agudeza visual mejor de 20/40, independientemente de si presentó 0 no complicaciones transoperatorias o postoperatorias. Ninguno de los pacientes tuvo visión peor a 20/100. Afortunadamente, la mayoría de las complicaciones pueden resolverse durante la cirugía, sin repercutir en la agudeza visual para la corrección de errores refractivos. Han, et al. reportan que después de un seguimiento por 4 años de pacientes sometidos a cirugía refractiva SMILE, el $92 \%$ de pacientes presentan agudeza visual no corregida mejor o igual a 20/2025. En nuestro estudio, el $81.17 \%$ presentó agudeza visual no corregida mejor o igual a 20/20.

\section{Conclusión}

La cirugía refractiva es un procedimiento quirúrgico en constante evolución en el campo de la oftalmología. Cada vez, los pacientes optan más por este tratamiento quirúrgico debido a las implicaciones económicas en el uso de anteojos y lentes de contacto, además por estética facial y ventajas por el desuso de lentes aéreos. Actualmente se cuentan con diferentes técnicas quirúrgicas, incluyendo LASIK, PRK, femto-LASIK, uso de lentes intraoculares fáquicos y la cirugía tipo SMILE.

$\mathrm{La}$ incidencia de complicaciones transoperatorias y postoperatorias por cirugía refractiva SMILE es relativamente baja y al presentarse pueden resolverse sin repercusión en la agudeza visual final. La incidencia de nuestro hospital comparada con la de otros hospitales en otras partes del mundo no se correlaciona.

Deben llevarse a cabo más reportes de complicaciones sobre esta nueva técnica quirúrgica, ya que si bien se conoce que tiene adecuados índices de seguridad y de predictibilidad, las complicaciones quirúrgicas pueden suceder. Además, son pocos los estudios realizados para concretar las posibles complicaciones que se presentan con la cirugía SMILE.

\section{Limitaciones}

Las limitaciones de este estudio radican en que se trata de un estudio descriptivo. Las cirugías fueron realizadas por diferentes médicos en formación y no por solo un cirujano experimentado. La técnica quirúrgica varía ligeramente en los estudios publicados en la literatura.

\section{Conflicto de intereses}

Los autores declaran la inexistencia de conflicto de intereses.

\section{Responsabilidades éticas}

Número de autorización del comité de ética, 2016CR1.

Protección de personas y animales. Los autores declaran que para esta investigación no se han realizado experimentos en seres humanos ni en animales.

Confidencialidad de los datos. Los autores declaran que han seguido los protocolos de su centro de trabajo sobre la publicación de datos de pacientes.

Derecho a la privacidad y consentimiento informado. Los autores declaran que en este artículo no aparecen datos de pacientes.

\section{Bibliografía}

1. Mysore N, Krueger R. Advances in Refractive Surgery May 2013 to June 2014. Asia Pac J Ophtalmol. 2015;4:2:112-20.

2. Chansue E, Tanehsakdi M, Swasdibutra S, McAlinden C. Efficacy, predictability and safety of small incisión lenticule extraction (SMILE). Eye Vis. 2015;2:14.

3. Aristeidou A, Taniguchi EV, Tsatsos M, Muller R, McAlinden C, Pineda R, et al. The evolution of corneal and refractive surgery with the femtosecond laser. Eye Vis. 2015;2:12.

4. Reinstein DZ, Archer TJ, Gobbe M, Johnson N. Accuracy and reproducibility of Artemis central flap thickness and visual outcomes of LASIK with the Carl zeiss meditec VisuMax femtosecond laser and MEL 80 excimer laser platforms. J Refract Surg. 2010;26:107-19.

5. Reinstein DR, Archer TJ, Gobbe M. Small incision lenticule extraction (SMILE) history, fundamentals of a new refractive surgery technique and clinical outcomes. Eye Vis. 2014;1:3.

6. Ivarsen A, Asp S, Hjortdal J. Safety and Complications of More Than 1500 small incision lenticule extraction procedures. Am Acad Ophtalmol. 2014;121:4.

7. Wang B, Naidu RK, Chu R, Dai J, Qu X, Zhou H. Dry eye disease following refractive surgery: $A$ 12-month follow-up of SMILE versus FS-LASIK in high myopia. J Ophtalmol. 2015; 2015:132417.

8. Dong Z, Zhou X. Irregular astigmatism after femtosecond laser refractive lenticule extraction. J Cataract Refract Surg. 2013;39:952-4

9. Reinstein DZ, Archer TJ, Dickeson Zl, Gobbe M. Trans-epithelial phototherapeutic keratectomy protocol for treating irregular astigmatism based population on epithelial thickness measurements by Artemis very high-frequency digital ultrasound. J Refract Surg. 2014;30:380-7.

10. Ekktet Chansue, Morakot Tenehsakdi, Sukanda Swasdibutra, Colm McAlinden. Safety and efficacy of VisuMax circle patterns for flap creation and enhancement following small incision lenticule extraction. Eye Vis. 2015;2:21. 
11. Marcus Blum, Kathrin Täubig, Christin Gruhn, Walter Sekundo, Kathleen $S$ Skunert. Five-year results of small incision lenticule extraction (SMILE). Ophtalmol. 2016;0:1-4.

12. Moshirfar M, McCaughey MV, Reinstein DZ, Shah R, Santiago-Caban L, Fenzl CR. Small-incision lenticule extraction. Cataract Refract Surg. 2015;41:652-65.

13. Wang Y, Ma J, Zhang J, Dou R, Zhang H, Li L,et al. Incidence and management of intraoperative complications during small-incision lenticule extraction in 3004 cases. J Cataract Refract Surg. 2017;43(6): 796-802.

14. Wong CW, Chan C, Tan D, Mehta JS. Incidence and management of suction loss in refractive lenticule extraction. J Cataract Refract Surg. 2014;40(12):2002-10.

15. Osman IM, Awad R, Shi W, Abou Shousha M. Suction loss during femtosecond laser-assisted small-incision lenticule extraction: Incidence and analysis of risk factors. J Cataract Refract Surg. 2016;42:246-50.

16. Ivarsen A, Sven A, Hjortdal J. Safety and Complications of More Than 1500 small incision lenticule extraction procedures. Ophtalmology. 2014; 121:4.

17. Ramirez-Miranda A, Ramirez-Luquin T, Navas A, Graue-Hernández EO. Refractive Lenticule Extraction Complications. Cornea. 2015;34(Suppl):S65-7.
18. Hamed AM, Abdelwahab SM, Soliman TT. Intraoperative complications of refractive small incision lenticule extraction in the early learning curve. Clin Ophthalmol. 2018;12:665-8.

19. Dong Z, Zhou X. Irregular astigmatism after femtosecond laser refractive lenticule extraction. J Cataract Refract Surg. 2013;39:952-4

20. Zhao J, He L, Yao P, Shen Y, Zhou Z, Miao H, et al. Diffuse lamellar keratitis after small-incision lenticule extraction. J Cataract Refract Surg. 2015;41:400-7

21. Gritz DC. LASIK interface keratitis: epidemiology, diagnosis and care. Curr Opin Ophtalmol. 2011;22:251-5.

22. Qiu PJ, Yang YB. Early changes to dry eye and ocularsurface after small-incision lenticule extraction for myopia. Int J Ophtalmol. 2016; 9:575-9.

23. Chehaibou I, Sandali O, Ameline B, Bouheraoua N, Borderie V, Laroche L. Bilateral infectious keratitis after small-incision lenticule extraction. J Cataract Refract Surg. 2016;42:626-30.

24. Llovet F, de Rojas V, Interlandi E, Martin C, Cobo-Soriano R, Ortega-Usobiaga J, et al. Infectious keratitis in 204,586 LASIK procedures. Ophtalmology. 2010;117:232-238.e1-4.

25. Han T, Zheng K, Chen Y, Gao Y, He L, Zhou X. Four-year observation of predictability and stability of small incision lenticule extraction. Ophthalmology. 2016;16:149. 